\title{
Avaliação da resposta de pacientes asmáticos com refluxo gastroesofágico após terapia com pantoprazol: estudo prospectivo, randomizado, duplo cego e placebo-controlado*
}

\author{
Evaluation of pantoprazol treatment response of patients with asthma and \\ gastroesophageal reflux: a randomized prospective \\ double-blind placebo-controlled study

\begin{abstract}
Leandro Heusi dos Santos ${ }^{1}$, lana Oliveira e Silva Ribeiroํㅜ, Pablo Gerardo Sánchez', Jorge Lima Hetzel ${ }^{2}$, José Carlos Felicetti ${ }^{3}$, Paulo Francisco Guerreiro Cardoso ${ }^{4}$
\end{abstract}

\begin{abstract}
Resumo
Objetivos: Determinar o efeito do tratamento da DRGE no controle clinico e funcional respiratório em indivíduos asmáticos e avaliar as características clinicas deste grupo de pacientes. Métodos: Pacientes com asma portadores de DRGE patológico documentado por pHmetria de $24 \mathrm{~h}$ foram avaliados por meio de questionários sobre sintomas respiratórios, digestivos, de qualidade de vida além de manometria esofágica, espirometria e pico de fluxo expiratório antes e após o estudo. Quarenta e nove indivíduos que apresentavam DRGE patológico na pHmetria esofágica de $24 \mathrm{~h}$ foram selecionados e participaram de ensaio clínico terapêutico com pantoprazol (40 mg/dia) controlado com placebo, randomizado, duplo-cego, paralelo por 12 semanas consecutivas. Resultados: Quarenta e quatro indivíduos completaram o estudo ( $n=22$ cada grupo). Houve melhora significativa no escore de sintomas respiratórios e na qualidade de vida somente no grupo que utilizou pantoprazol ( $p=0,01$ e $p=0,001$, respectivamente). Os parâmetros funcionais respiratórios não se modificaram com os diferentes tratamentos. Conclusões: Neste estudo, o tratamento efetivo do RGE melhorou a qualidade de vida, determinou diminuição dos sintomas em asma de maneira significativa no grupo que utilizou medicamento, contudo sem alterar os parâmetros funcionais.
\end{abstract}

Descritores: Asma; Refluxo gastroesofágico; Tratamento; Diagnóstico; Antiácidos.

\begin{abstract}
Objectives: To determine the effect that the treatment of GERD has on the clinical management, as well as the respiratory function, of patients with asthma and to evaluate the clinical characteristics of this group of patients. Methods: Patients with asthma and concomitant GERD, documented using $24 \mathrm{~h} \mathrm{pH}$-metry, were evaluated by means of quality of life questionnaires, as well as questionnaires related to respiratory and digestive symptoms. In addition, esophageal manometry, spirometry and the determination of peak expiratory flow were also performed prior to and after the study. Forty-nine individuals who were diagnosed with GERD by means of $24 \mathrm{~h}$ esophageal pH-metry were selected and participated in a clinical randomized double-blind placebo-controlled study, involving the administration of $40 \mathrm{mg} / \mathrm{day}$ of pantoprazol for 12 consecutive weeks. Results: Forty-four individuals completed the study ( $\mathrm{n}=22$ per group). There was significant improvement in the scores for respiratory symptoms and quality of life only in the group that received pantoprazol $(p=0.01$ and $p=0.001$, respectively). No respiratory function parameters changed in either group. Conclusions: In this study, the effective treatment of GERD improved patient quality of life, and the symptoms of asthma significantly decreased in the group that received the medication. There were no changes in pulmonary function parameters.
\end{abstract}

Keywords: Asthma; Gastroesophageal reflux/treatment; Diagnosis; Antacids.

\footnotetext{
* Trabalho realizado nos Serviços de Pneumologia, Cirurgia Torácica e Laboratório de Função Esofagiana do Pavilhão Pereira Filho, Santa Casa de Porto Alegre, RS, em colaboração com o Programa de Pós-graduação em Pneumologia da Universidade Federal do Rio Grande do Sul e com a Fundação Faculdade Federal de Ciências Médicas de Porto Alegre - FFFCMPA - Porto Alegre (RS) Brasil.

1. Mestre em Pneumologia pela Universidade Federal do Rio Grande do Sul (RS) Brasil.

2. Professor Associado, Disciplina de Pneumologia da Fundação Faculdade Federal de Ciências Médicas de Porto Alegre (RS) Brasil.

3. Professor assistente na Disciplina de Cirurgia Torácica da Fundação Faculdade Federal de Ciências Médicas de Porto Alegre (RS) Brasil.

4. Professor Associado na Disciplina de Cirurgia Torácica da Fundação Faculdade Federal de Ciências Médicas de Porto Alegre (RS) Brasil.

Endereço para correspondência: Paulo Francisco Guerreiro Cardoso. Santa Casa de Porto Alegre - Pavilhão Pereira Filho, Rua Professor Annes Dias, 285, $1^{\circ}$ andar, PPF, CEP 90020-090, Porto Alegre, RS, Brasil.

Tel 5551 3227-3909, Fax 5551 3228-2510. E-mail: cardosop@gmail.com

Recebido para publicação em 05/08/2005. Aprovado, após revisão, em 20/06/2006
} 


\section{Introdução}

A prevalência de doenças acometendo o sistema respiratório e digestivo, em forma conjunta, é elevada. ${ }^{(1)}$ Estima-se que a asma acometa 10\% da população adulta. ${ }^{(2)}$ Por outro lado, em estudos epidemiológicos, o relato de pirose ocasional chega a 58\% e o de pirose diária em até 7\% dos adultos. $^{(3)}$ Nas últimas décadas, avolumaram-se os estudos associando a doença do refluxo gastresofágico (DRGE) a manifestações respiratórias e otorrinolaringológicas. ${ }^{(4-7)}$ Foram descritos mecanismos fisiopatológicos bem documentados que ajudaram a explicar como estas doenças interagiam. Estudos em animais ${ }^{(8,9)}$ e em humanos ${ }^{(10,11)}$ demonstraram que a DRGE poderia agravar a asma por microaspiração, reflexo vagal e aumento da responsividade das vias aéreas. Assim sendo, a terapia anti-refluxo, tanto medicamentosa quanto cirúrgica, deveria melhorar ou, eventualmente, resolver os sintomas respiratórios em alguns pacientes. Entretanto, metanálises publicadas em $1998^{(11)} \mathrm{e}$ em $2000^{(12)}$ reunindo estudos sobre a terapia antirefluxo em pacientes asmáticos, demonstraram que a melhora objetiva dos sintomas respiratórios dos pacientes era acompanhada de melhora apenas discreta ou de nenhuma melhora mensurável na função pulmonar. Em uma outra revisão sistemática concluiu-se que o tratamento da DRGE não resultou em qualquer benefício consistente para pacientes asmáticos. ${ }^{(13)} 0$ papel da DRGE como agravante de asma permanece controverso, apesar da reconhecida associação entre estas duas doenças. 0 mesmo ocorre em relação ao tratamento do refluxo em asmáticos.

0 presente estudo procura analisar de forma sistemática e prospectiva a resposta clínica/ funcional e a qualidade de vida dos asmáticos portadores de refluxo gastresofágico patológico à terapia anti-refluxo por ablação ácida farmacológica com um bloqueador de bomba protônica (pantoprazol, $40 \mathrm{mg} / \mathrm{dia}$ ) por um período de três meses consecutivos.

\section{Métodos}

Foi realizado um estudo prospectivo, randomizado, duplo cego paralelo e controlado por placebo, com um período de 90 dias de acompanhamento. Após a aprovação do estudo pelo Comitê de Ética em Pesquisa da Santa Casa de Porto Alegre, foram inclu- ídos pacientes maiores de 18 anos, com diagnóstico clínico e funcional de asma associada à DRGE. Em relação à asma, os pacientes deveriam apresentar história clínica compatível e doença estável por mais de dois meses, bem como espirometria característica (relação entre volume expiratório forçado no primeiro segundo e capacidade vital forçada $\left[\mathrm{VEF}_{1} /\right.$ CVF] $<90 \%$ do previsto, traduzindo obstrução ao fluxo aéreo e reversibilidade da obstrução dada por um $\mathrm{VEF}_{1}>200 \mathrm{~mL}$ e 7\% do previsto) ou hiperresponsividade brônquica positiva à metacolina, no teste de broncoprovocação. Para a DRGE sintomática ou não, o diagnóstico foi confirmado por pHmetria esofágica de $24 \mathrm{~h}$ precedida por esofagomanometria estacionária. Os critérios de exclusão foram: história recente de tabagismo (últimas oito semanas); anormalidade ao exame radiológico dos seios da face/tórax; história de uso de bloqueadores de bomba de prótons até quatro semanas ou de bloqueadores de receptor $\mathrm{H}-2$ até duas semanas antes da inclusão no estudo; hipertensão arterial sistêmica quando em uso de inibidores da enzima de conversão de angiotensina, bloqueadores do receptor $\beta$-adrenérgico ou bloqueadores de canal de cálcio que não pudessem ser trocados; presença de outra doença sistêmica grave; gravidez; analfabetismo ou total falta de compreensão para o preenchimento dos formulários.

As provas de função pulmonar foram realizadas com um espirômetro de fluxo KOKO ${ }^{\circledR}$ (Pulmonary Data Service Instrumentation, Inc., Louisville, KY, EUA). A técnica do exame foi realizada conforme as Diretrizes para Testes de Função Pulmonar. ${ }^{(14)}$

A esofagomanometria foi realizada com cateter perfundido de 6 canais de pressão (Synectics, Estocolmo, Suécia) com 3 canais radiais distais e 3 proximais distando $5 \mathrm{~cm}$. 0 cateter era perfundido com bomba pneumohidráulica capilar (Mui Scientific, Mississauga, Ontário, Canadá) e a pressão analisada por polígrafo digital (Polygraph ${ }^{\circledR}$; Synectics) com leitura das pressões em tempo real (Polygram ${ }^{\circledR}$; Synectics). Imediatamente após a esofagomanometria, um cateter de pHmetria com um eletrodo distal semi-descartável de antimônio (Synectics) foi passado por via nasoesofagiana, sendo posicionado $5 \mathrm{~cm}$ acima do limite superior do esfíncter esofagiano inferior, o qual fora previamente localizado pela manometria. 0 eletrodo era conectado a um aparelho portátil (Mk III; Synectics), com o qual o paciente permanecia por $24 \mathrm{~h}$. Ao retirar-se o cateter 
de pHmetria, os dados eram transferidos para o programa de análise (Esophogram ${ }^{\circledR}$; Synectics). Os parâmetros analisados e o escore utilizado foram os de Johnson \& DeMeester, ${ }^{(15)}$ cujos valores normais encontram-se na Tabela 1.

Os pacientes selecionados passaram por uma fase de pré-terapia, na qual realizavam diariamente, pela manhã e a noite e por 10 dias consecutivos, a manobra do pico do fluxo expiratório (PFE), com um medidor da marca Mini-Wright ${ }^{\circledR}$ (Clement Clarke International Limited, Essex, Reino Unido).
Preenchiam, também diariamente e por 10 dias seguidos, um diário de sintomas de asma e de DRGE, o qual foi baseado no diário de sintomas de Harding et al., ${ }^{(16)}$ mostrado na Tabela 1. Ainda nesta fase, revisou-se a técnica de utilização da medicação inalatória prévia, bem como a adesão ao tratamento.

Ao término desta fase, foi aplicado o questionário de qualidade de vida em asma (Unifesp - EPM) ${ }^{(16,17)}$, o qual foi baseado nas áreas de domínio investigadas por Juniper e Guyatt, ${ }^{(18)}$ sendo então adaptado

Tabela 1 - Características demográficas, clínicas e funcionais dos pacientes no inicio do estudo.

\begin{tabular}{|c|c|c|c|c|c|}
\hline \multirow[t]{2}{*}{ Variável } & \multicolumn{2}{|c|}{ Placebo } & \multicolumn{2}{|c|}{ Medicamento } & \multirow[t]{2}{*}{$\mathrm{p}$} \\
\hline & $\mathrm{n}$ & $\%$ & $\mathrm{n}$ & $\%$ & \\
\hline ldade (anos) & $45 \pm 12$ & - & $40 \pm 12$ & - & 0,15 \\
\hline Homens & 2 & 9,1 & 7 & 36,4 & 0,007 \\
\hline Uso de $\beta_{2}$ de longa ação & 14 & 64 & 10 & 45 & 0,36 \\
\hline Uso de corticóide oral & 4 & 18 & 2 & 9 & 0,66 \\
\hline \multicolumn{6}{|l|}{ pHmetria } \\
\hline -Escore DeMeester (normal 14,7) & $40,1 \pm 28$ & - & $29,7 \pm 12$ & 0,12 & 0,12 \\
\hline -\% tempo total (normal <4,2\%) & $9,5 \pm 7$ & - & $7,5 \pm 3$ & 0,25 & 0,25 \\
\hline -\% tempo ortostatismo (normal 6,3\%) & $8,3 \pm 7$ & - & $7,2 \pm 5$ & 0,58 & 0,58 \\
\hline -\% tempo supino (normal <1,2\%) & $11,7 \pm 14$ & - & $7,7 \pm 8$ & 0,23 & 0,23 \\
\hline -Episódios > 5 min $($ normal <3) & $4,7 \pm 4$ & - & $3 \pm 2$ & 0,16 & 0,16 \\
\hline - Nºpisódios refluxo (normal <50) & $115,9 \pm 59$ & - & $94 \pm 37$ & 0,12 & 0,12 \\
\hline \multicolumn{6}{|l|}{ Manometria* } \\
\hline -Tônus EEl (normal 14-40 mmHg) & $15,5 \pm 5$ & - & $18,1 \pm 5$ & 0,06 & 0,06 \\
\hline -Extensão EEl & $5 \pm 1$ & - & $5,5 \pm 1$ & 0,16 & 0,16 \\
\hline -Tônus EES & $67,6 \pm 32$ & - & $63,4 \pm 31$ & 0,65 & 0,65 \\
\hline ES noturno asma* & $66 \pm 26$ & - & $67 \pm 27$ & - & 0,91 \\
\hline ES diurno asma* & $68,8 \pm 26$ & - & $68,4 \pm 29$ & - & 0,96 \\
\hline \multicolumn{6}{|l|}{$\mathrm{QV}^{*}$} \\
\hline -Total & $63,8 \pm 13$ & - & $61,6 \pm 15$ & 0,63 & 0,91 \\
\hline -Limitação física & $60 \pm 20$ & - & $63,7 \pm 15$ & 0,59 & 0,96 \\
\hline -Sintomas & $47,3 \pm 23$ & - & $55,4 \pm 26$ & 0,42 & 0,63 \\
\hline -Adesão & $47,8 \pm 26$ & - & $53,6 \pm 24$ & 0,45 & 0,59 \\
\hline -Socioeconômica & $61,3 \pm 19$ & - & $60,4 \pm 20$ & 0,87 & 0,42 \\
\hline -Psicossocial & $56 \pm 13$ & - & $58 \pm 22$ & 0,77 & 0,45 \\
\hline PFE diurno* & $264 \pm 86$ & - & $317 \pm 13$ & - & 0,11 \\
\hline PFE noturno* & $261 \pm 83$ & - & $307 \pm 12$ & - & 0,14 \\
\hline CVF (\% previsto)* & $85,8 \pm 15$ & - & $79,5 \pm 17$ & - & 0,20 \\
\hline $\operatorname{VEF}_{1}(\%$ previsto)* & $60,4 \pm 19$ & - & $61,6 \pm 19$ & - & 0,83 \\
\hline $\mathrm{VEF}_{1} / \mathrm{CVF}$ (\% previsto)* & $69,4 \pm 13$ & - & $71,7 \pm 11$ & - & 0,53 \\
\hline
\end{tabular}

*EEl: esfíncter esofagiano inferior; EES: esfíncter esofagiano superior; ES: escore de sintomas; PFE: pico de fluxo expiratório; CVF: capacidade vital forçada; $\mathrm{VEF}_{1}$ : volume expiratório forçado no primeiro segundo; QV: escore qualidade de vida, total em pontos; Media \pm Desvio Padrão. 
e validado à população brasileira. Ao final deste período, iniciou-se a intervenção. Os pacientes foram aleatoriamente distribuídos em dois grupos de tratamento, dos quais tanto o paciente como os examinadores desconheciam a medicação utilizada. Um dos grupos recebeu pantoprazol, $40 \mathrm{mg}$ em dose única diária, e o outro grupo recebeu placebo. Todos os indivíduos participantes foram avaliados mensalmente, por meio de um questionário de consulta para avaliação da aderência ao tratamento e/ou possíveis efeitos colaterais. Durante a última semana do estudo uma nova pHmetria de $24 \mathrm{~h}$ de controle foi realizada. Na consulta final, o questionário de qualidade de vida foi reaplicado, o diário de sintomas preenchido por 10 dias foi coletado, assim como houve a realização do PFE e a reavaliação da função pulmonar através de espirometria.

Os dados foram armazenados em planilha eletrônica (Microsoft Office Excel ${ }^{\circledR}$ ) e as análises foram realizadas com o auxílio do programa estatístico SPSS ${ }^{\circledR} 10$ (SPSS Inc., Chicago, ll, EUA). As variáveis demográficas, clínicas e laboratoriais de ambos os grupos foram avaliadas para distribuição normal, sendo expressas em média e desvio padrão. Para avaliar as diferenças entre as variáveis de $\mathrm{pHmetria,}$ escores de sintomas e de qualidade de vida de cada grupo entre os períodos antes e após o tratamento, foi utilizado o teste de Wilcoxon de postos assinalados. As mesmas variáveis foram comparadas entre os dois grupos, antes e após o tratamento, pelo teste de Mann-Whitney. A comparação dos PFE matinal e noturno e dos valores espirométricos entre os grupos tratamento e placebo foi feita com o teste $t$ de Student não pareado. Para a comparação entre cada grupo, nos momentos antes e depois do estudo, foi utilizado o teste t de Student pareado. Para todos os testes estatísticos, o nível de significância adotado foi de $5 \%(p<0,05)$.

\section{Resultados}

Dos 73 pacientes asmáticos que preencheram os critérios de entrada no estudo, 49 apresentaram DRGE e foram elegíveis para o estudo. Destes, cinco foram excluídos: dois por piora dos seus sintomas, necessitando internação hospitalar para tratamento; um por abandono do protocolo; um por intolerância à medicação utilizada no estudo; e um por iniciar o hábito tabágico. Assim, um total de 44 pacientes participou do estudo. Destes, 35 completaram o protocolo integralmente, uma vez que 9 pacientes não realizaram a pHmetria ao final do estudo. Não obstante, estes também foram incluídos na análise, totalizando desta forma 22 pacientes em cada grupo. Os grupos foram denominados de 'm' (pacientes que receberam a substância ativa) e 'p' (para os pacientes que receberam placebo). As características gerais dos pacientes estão descritas na Tabela 1. Não observamos diferenças entre os grupos no início do estudo em relação às variáveis clínicas de asma e DRGE, bem como aos escores de qualidade de vida e função pulmonar.

Neste estudo houve um número maior de mulheres com sintomas respiratórios crônicos e predomínio de doença moderada/grave, visto a quantidade de medicação $\beta_{2}$-agonista de longa duração e do uso de corticóide via oral necessários para controle de seus sintomas. Da mesma forma, estas apresentavam DRGE com altos níveis de exposição ácida esofágica com predominância no periodo noturno, estando no grupo placebo as com as maiores alterações. Do total de pacientes estudados, 9 (20\%) eram assintomáticos em relação à DRGE. Quanto aos achados sobre o controle da DRGE em ambos os grupos, houve uma expressiva melhora tanto no escore de sintomas como nos índices da pHmetria no grupo tratamento, sendo que apenas um único paciente deste grupo não teve normalizado seu escore de DeMeester após os 3 meses do estudo. Houve uma melhora no escore de sintomas em ambos os grupos isoladamente, porém de maneira estatisticamente significativa no grupo 'm'. Contudo, as diferenças entre os grupos não foram significativas quando avaliadas ao final do estudo. Em relação à qualidade de vida, o grupo ' $m$ ' obteve melhora significante em alguns dos domínios do escore de qualidade de vida e, quando comparados os grupos ao final da intervenção, apenas o escore global mostrou diferença significativamente estatística. Porém, houve uma tendência para melhora no grupo ' $m$ ' em todos os domínios (Tabela 2). As respostas nas provas funcionais de ambos os grupos são mostradas na Tabela 3.

No que tange à presença de sintomas respiratórios associados a sintomas de refluxo (SRAR), dos 44 pacientes, 37 foram divididos em 2 grupos denominados 'SRAR-positivo' e 'SRAR-negativo', sendo que os outros 7 pacientes foram excluídos devido a dados incompletos para esta análise. A Tabela 4 descreve as características iniciais destes 
Tabela 2 - Comparação entre as variáveis de controle clínico da asma e qualidade de vida entre o inicio e o fim do estudo, para cada grupo e entre os dois grupos ao final do estudo.

\begin{tabular}{|c|c|c|c|c|c|c|c|}
\hline \multirow[t]{2}{*}{ Variável } & \multicolumn{3}{|c|}{ Placebo } & \multicolumn{3}{|c|}{ Medicamento } & \multirow[t]{2}{*}{ p entre grupos } \\
\hline & Inicial & Final & $p$ & Inicial & Final & $p$ & \\
\hline ES diurno* & $68,8 \pm 26$ & $64,92 \pm 4$ & 0,29 & $69,2 \pm 29$ & $58,9 \pm 23$ & 0,01 & 0,11 \\
\hline ES noturno & $66 \pm 25$ & $63,42 \pm 6$ & 0,24 & $66,92 \pm 7$ & $57,9 \pm 23$ & 0,01 & 0,16 \\
\hline QV total* & $63,8 \pm 13$ & $61,8 \pm 13$ & 0,25 & $61,61 \pm 5$ & $48,7 \pm 12$ & 0,00 & 0,001 \\
\hline QV limit. física & $60 \pm 20$ & $58,1 \pm 18$ & 0,31 & $63,7 \pm 15$ & $52,81 \pm 7$ & 0,02 & 0,67 \\
\hline QV Sintomas & $47,3 \pm 23$ & $53,4 \pm 24$ & 0,18 & $55,42 \pm 6$ & $40,8 \pm 15$ & 0,05 & 0,08 \\
\hline QV Adesão & $47,8 \pm 26$ & $42,22 \pm 6$ & 0,46 & $53,62 \pm 4$ & $37,42 \pm 7$ & 0,08 & 0,55 \\
\hline QV Socio econ. & $61,31 \pm 9$ & $59,72 \pm 1$ & 0,62 & $60,4 \pm 20$ & $56,31 \pm 9$ & 0,39 & 0,58 \\
\hline QV Psicossocial & $56 \pm 13$ & $51,61 \pm 7$ & 0,33 & $58 \pm 22$ & $43,62 \pm 3$ & 0,03 & 0,11 \\
\hline
\end{tabular}

*ES: escore de sintomas; QV: qualidade de vida, em pontos; Valores representados = média \pm desvio padrão.

2 grupos, não se observando diferenças significativas. Posteriormente, com o intuito de verificar se a característica SRAR seria um fator preditor de melhora dos parâmetros respiratórios, avaliamos somente o grupo positivo para esta característica, estudando comparativamente antes e após a intervenção terapêutica e também a comparação entre os dois grupos. Não houve melhora nos valores funcionais respiratórios em ambos os grupos estudados tanto na análise $\mathrm{dm}$ cada grupo, tampouco quando comparados ao final do estudo (Tabela 5).

\section{Discussão}

0 presente estudo avaliou a terapia para a DRGE ao longo de três meses em pacientes asmáticos, de maneira randomizada e controlada por placebo. A correlação entre estas duas doenças vem sendo amplamente estudada na literatura através de ensaios clínicos avaliando o papel do tratamento da DRGE na melhora da asma. ${ }^{(16,19-21)}$ Não obstante os resultados, permanecem as controvérsias sobre o tema. Nos últimos anos, duas grandes revisões foram publicadas acerca do impacto do tratamento da DRGE sobre o controle da asma. Na primeira, ${ }^{(11)}$ concluiu-se que o tratamento da DRGE melhora os sintomas em 69\% dos casos, reduz o uso da medicação para asma em 62\%, além de determinar melhora no pico de fluxo vespertino em $26 \%$ destes pacientes. Na segunda, ${ }^{(13)}$ concluiu-se que o tratamento de DRGE não melhora consistentemente os sintomas da asma, não reduz o uso de medicações nem possui impacto significativo na função pulmonar. Contudo, os próprios autores reconheceram as limitações metodológicas dos estudos incluídos nestas revisões, ressaltando a necessidade de outros ensaios clínicos voltados a esta questão. Um dos pontos controversos descritos por estes autores é de que, dos ensaios clínicos estudados, apenas seis utilizaram inibidores de bomba protônica. Ademais, estes estudos demonstraram que pacientes com manifestações respiratórias da DRGE necessitariam de doses maiores de um inibidor de bomba de prótons para o controle de

Tabela 3 - Comparação entre as variáveis funcionais respiratórias entre o início e o fim do estudo, para cada grupo e entre os 2 grupos ao final do estudo.

\begin{tabular}{|c|c|c|c|c|c|c|c|}
\hline \multirow[t]{2}{*}{ Variável } & \multicolumn{3}{|c|}{ Placebo } & \multicolumn{3}{|c|}{ Medicamento } & \multirow[t]{2}{*}{ p entre grupos } \\
\hline & inicial & final & $\mathrm{p}$ & inicial & final & $p$ & \\
\hline CVF (\% previsto)* & $85,81 \pm 5$ & $85,9 \pm 16$ & 0,57 & $79,5 \pm 17$ & $81,3 \pm 18$ & 0,79 & 0,40 \\
\hline $\operatorname{VEF}_{1}\left(\%\right.$ previsto) ${ }^{*}$ & $60,4 \pm 19$ & $58,9 \pm 13$ & 0,55 & $61,6 \pm 19$ & $62 \pm 21$ & 0,46 & 0,65 \\
\hline $\mathrm{VEF}_{1} / \mathrm{CVF}$ (\% previsto) & $69,4 \pm 13$ & $70,2 \pm 12$ & 0,16 & $71,7 \pm 11$ & $73,8 \pm 14$ & 0,54 & 0,40 \\
\hline PFE diurno (L/min)* & $264 \pm 86$ & $267 \pm 81$ & 0,59 & $317 \pm 126$ & $327 \pm 77$ & 0,23 & 0,74 \\
\hline PFE noturno (L/min) & $261 \pm 83$ & $269 \pm 77$ & 0,39 & $307 \pm 121$ & $323 \pm 127$ & 0,46 & 0,10 \\
\hline
\end{tabular}

*PFE: pico de fluxo expiratório; CVF: capacidade vital forçada; $\mathrm{VEF}_{1}$ : volume expiratório forçado no primeiro segundo; e valores representados = média \pm desvio padrão. 
Tabela 4 - Comparação entre as variáveis estudadas, entre os grupos com e sem sintomas respiratórios associados a sintomas de refluxo SRAR, no inicio do estudo.

\begin{tabular}{lccc}
\hline \multicolumn{1}{c}{ Variável } & SRAR positivo $(\mathrm{n}=15)$ & SRAR negativo $(\mathrm{n}=22)$ & $\mathrm{p}$ \\
\hline ES asma noturno* $^{*}$ & $78 \pm 22$ & $58 \pm 25$ & 0,15 \\
ES asma diurno & $80 \pm 22$ & $59 \pm 26$ & 0,17 \\
VEF $_{1}$ (\% previsto)* & $66 \pm 24$ & $59 \pm 14$ & 0,31 \\
VEF $_{1} /$ CVF (\% previsto) & $75 \pm 11$ & $70 \pm 11$ & 0,19 \\
CVF (\% previsto)* & $87 \pm 11$ & $80 \pm 12$ & 0,24 \\
ES DRGE & $15 \pm 6$ & $11 \pm 9$ & 0,12 \\
PFE (diurno) & $314 \pm 90$ & $286 \pm 120$ & 0,43 \\
PFE (noturno) & $308 \pm 81$ & $287 \pm 111$ & 0,52 \\
Escore DeMeester & $30 \pm 11$ & $35 \pm 22$ & 0,36 \\
\% tempo ortostatismo & $8,1 \pm 3$ & $7,4 \pm 7$ & 0,67 \\
\% tempo supino & $6 \pm 6$ & $10 \pm 8$ & 0,12 \\
\% tempo total & $7,4 \pm 2,5$ & $9 \pm 6$ & 0,33 \\
\hline
\end{tabular}

*ES: escore de sintomas; CVF: capacidade vital forçada; $\mathrm{VEF}_{1}$ : volume expiratório forçado no primeiro segundo; PFE: pico de fluxo expiratório; QV: questionário de qualidade de vida, valores em pontos; e valores representados = média \pm desvio padrão.

seus sintomas. ${ }^{(22)}$ Com base nestes dados, optamos pela realização de nova pHmetria de $24 \mathrm{~h}$ ao final do estudo, com o intuito de verificar a real eficácia da supressão ácida instituída, objetivando facilitar a análise dos resultados.

No presente estudo houve uma supressão expressiva da acidez esofágica no grupo que utilizou medicação, uma vez que apenas um paciente não teve normalizado seu escore de DeMeester ${ }^{(15)}$ ao final do estudo. Estes achados poderiam ser explicados pelo uso de um inibidor de bomba de prótons de reconhecida eficácia e em doses adequadas ao longo do estudo. Outros autores utilizaram-se deste recurso para os mesmos fins e concluíram que a ablação ácida ao final do estudo era um fator de importância no contexto do tratamento e aferição de seus resultados. ${ }^{(22,23)}$

A população avaliada em nosso estudo constituiu-se de asmáticos em uso continuado de corticósteróides, sendo necessária em uma grande parcela destes pacientes a associação de $\beta_{2}$-agonistas de longa duração para o tratamento de sua doença. A despeito desta terapêutica, muitos apresentavam sintomas diurnos e noturnos alem de limitação da sua qualidade de vida, fatos estes que sugerem ser esta uma população de asmáticos com doença mais grave. Em poucos estudos na literatura comparando o uso de inibidores de bomba protônica com

Tabela 5 - Comparação entre variáveis estudadas, para o grupo SRAR positivo, entre o inicio e o fim do estudo e entre os grupos ao final do estudo.

\begin{tabular}{|c|c|c|c|c|c|c|c|}
\hline \multirow[t]{3}{*}{ Variáveis } & \multicolumn{7}{|c|}{ SRAR positivo $(n=15)$} \\
\hline & \multicolumn{3}{|c|}{ Placebo $(\mathrm{n}=8)$} & \multicolumn{3}{|c|}{ Medicamento $(\mathrm{n}=7)$} & \multirow{2}{*}{$\begin{array}{l}p \text { entre } \\
\text { grupos }\end{array}$} \\
\hline & Inicial & Final & $\mathrm{p}$ & Inicial & Final & $\mathrm{p}$ & \\
\hline ES asma diurno* & $90 \pm 16$ & $92 \pm 17$ & 0,30 & $80 \pm 21$ & $60 \pm 24$ & 0,05 & 0,02 \\
\hline ES asma noturno & $81 \pm 15$ & $80 \pm 14$ & 0,39 & $77 \pm 25$ & $59 \pm 27$ & 0,04 & 0,03 \\
\hline PFE diurno $(\mathrm{L} / \mathrm{min})^{*}$ & $284 \pm 98$ & $300 \pm 92$ & 0,66 & $323 \pm 86$ & $334 \pm 82$ & 0,47 & 0,62 \\
\hline PEF noturno (L/min) & $266 \pm 94$ & $311 \pm 90$ & 0,35 & $324 \pm 67$ & $320 \pm 72$ & 0,68 & 0,84 \\
\hline CVF $(\%)^{*}$ & $91 \pm 16$ & $94 \pm 16$ & 0,60 & $81 \pm 11$ & $85 \pm 19$ & 0,22 & 0,49 \\
\hline $\operatorname{VEF}_{1}(\%)^{*}$ & $64 \pm 23$ & $64 \pm 27$ & 0,29 & $68 \pm 17$ & $73 \pm 20$ & 0,11 & 0,53 \\
\hline QV global* & $60 \pm 19$ & $62 \pm 16$ & 0,33 & $62 \pm 8$ & $46 \pm 8$ & 0,01 & 0,01 \\
\hline
\end{tabular}

*SRAR: sintomas respiratórios associados a refluxo; ES: escore de sintomas; PFE: pico de fluxo expiratório; CVF: capacidade vital forçada; $\mathrm{VEF}_{1}$ : volume expiratório forçado no primeiro segundo; $\mathrm{QV}$; questionário de qualidade de vida, em total de pontos; valores representados = média \pm desvio padrão). 
placebo descreveu-se ou sugeriu-se a gravidade da doença respiratória. Um dos ensaios terapêuticos em que foi mencionada a gravidade da expressão clinica dos pacientes analisados tratava-se de um estudo aberto. ${ }^{(22)}$ Este fator torna-se importante, uma vez que a ausência da mensuração no inicio do protocolo poderia ser mais um fator de viés nestes estudos, já que pacientes com enfermidades mais graves são menos propensos a melhoras expressivas de suas doenças.

As características da manometria esofágica nestes pacientes demonstram que, em ambos os grupos no inicio do estudo, o tônus do esfíncter esofagiano inferior situava-se no limite inferior da normalidade. Tal achado coincide com os achados de outro estudo realizado previamente em nosso laboratório de função esofagiana, ${ }^{(24)}$ no qual a média do tônus do esfíncter esofagiano inferior também apresentavase no limite inferior da normalidade $(15,3 \mathrm{mmHg})$. Neste mesmo estudo, a análise do perfil motor esofágico de 164 pacientes asmáticos submetidos a esofagomanometria demonstrou a presença de alterações motoras em 32\% dos indivíduos, sendo em sua maioria representadas por hipomotilidade ou motilidade não efetiva no esôfago distal. Em estudo semelhante, ${ }^{(25)}$ também descreveu-se uma incidência elevada de alterações manométricas em pacientes asmáticos quando comparados a um grupo controle, sendo a motilidade esofagiana não efetiva o distúrbio mais comum em 53\% dos pacientes. Em ambos os estudos, os autores sugerem que a associação de anormalidade na pHmetria e dismotilidade esofágica pode ser indicativa de microaspiração de ácido neste grupo como fator desencadeante, agravante ou mantenedor dos sintomas respiratórios.

Em concordância com outros estudos descritos na literatura, ${ }^{(26,27)}$ nossa análise não demonstrou mudanças na função respiratória dos pacientes tratados com ablação ácida. Este paradoxo foi encontrado também em outros estudos em que o tratamento clínico ou mesmo cirúrgico da DRGE melhorara os sintomas de asma a despeito de não demonstrarem melhora da função pulmonar. Eventualmente, as limitações já descritas neste e em outros estudos, tais como o número reduzido de pacientes ou mesmo um tempo de ablação ácida demasiadamente curto, poderiam ter contribuído para tais resultados. Estes achados também poderiam ser explicados por evidências que demonstram um aumento da ventilação-minuto como fator desencadeante de dispnéia e de desconforto torácico em pacientes submetidos a perfusão ácida esofágica sem, contudo, haver obstrução das vias aéreas. ${ }^{(28)}$

Field et al. ${ }^{(28)}$ buscaram, por meio de seu questionário de DRGE e asma, a presença de SRAR. Posteriormente, Harding et al. ${ }^{(16)}$ identificaram essa condição como fator preditivo de resposta ao tratamento. Atualmente, acredita-se que este grupo de pacientes, asmáticos portadores de DRGE que reportam sintomas respiratórios diretamente relacionados ao refluxo, responda melhor às medidas anti-refluxo e ao tratamento com medicamentos, obtendo conseqüentemente um melhor controle da asma quando tratados para a DRGE. Em nosso estudo, também avaliamos a presença de SRAR através de questionário, sendo que a maioria não apresentou este fator. Foram também comparadas algumas características iniciais dos pacientes, dividindo-os em relação à presença ou não de SRAR, no intuito de melhor definir este grupo de asmáticos, sem encontrarem-se quaisquer diferenças significativas. Entretanto, quando apenas os pacientes com SRAR foram observados em relação aos desfechos do estudo, encontramos novamente uma diferença significativa na melhora do escore de sintomas e na qualidade de vida no grupo que utilizou medicamento. Sendo assim, poderíamos inferir que a pesquisa desta característica em pacientes asmáticos portadores de DRGE sintomática possa ser um subsídio importante na indicação da terapia para este grupo.

Por ser este um estudo prospectivo, randomizado, duplo-cego e de metodologia complexa, algumas deficiências e limitações na sua realização devem ser mencionadas. 0 tamanho final da amostra foi pequeno, podendo assim ter contribuído para a ausência de achados mais contundentes nos desfechos analisados. Em ensaios clínicos, em que a amostra de pacientes foi maior, como o de Kiljander et al., ${ }^{(19)}$ que estudou 57 pacientes de forma controlada e cruzada, verificou-se uma melhora funcional de 20\% em 35\% dos pacientes tratados. Ademais, o tempo de seguimento de três meses pode ter sido curto para a verificação de melhora consistente nos parâmetros funcionais respiratórios destes pacientes.

Na medida em que se avança no conhecimento das manifestações extra-esofágicas da DRGE, cresce a necessidade de empreender-se um estudo amplo de prevalência da DRGE avaliando asmáticos em 
uma população não referenciada, ou seja, livre dos vícios de seleção, e ainda dispondo da confirmação objetiva da presença de exposição esofágica anormal ao ácido gástrico neste grupo de pacientes. Alem disso, há evidências suficientes que indicam que muito há que se fazer no âmbito da intervenção terapêutica neste grupo de pacientes. Torna-se imperativo que se estabeleçam quais as características destes pacientes asmáticos que serviriam como preditores de resposta à terapia da DRGE, assim como os parâmetros mais adequados para a mensuração desta. Concluímos portanto que, no presente estudo, o tratamento da DRGE melhorou significativamente a qualidade de vida e o índice de sintomas dos pacientes asmáticos sem, no entanto, alterar significativamente os parâmetros funcionais respiratórios. Dentre os pacientes estudados, aqueles que possuíam sintomas respiratórios associados ao refluxo e que foram submetidos à ablação ácida farmacológica foram os que apresentaram redução objetiva no escore de asma e melhora na qualidade de vida ao final do período de avaliação.

\section{Agradecimentos}

Os autores agradecem à Altana Pharma, à Pós-graduação em Pneumologia da Universidade Federal do Rio Grande do Sul e à Santa Casa de Porto Alegre.

\section{Referências}

1. Napierkowski J, Wong RK. Extraesophageal manifestations of GERD. Am J Med Sci. 2003;326(5):285-99.

2. Sociedade Brasileira de Pneumologia e Tisiologia. 111 Consenso Brasileiro no Manejo da Asma. Jornal de Pneumol. 2002;28(Supl 1):110-20.

3. JohansonJF.Epidemiologyofesophagealand supraesophageal reflux injures. Am J Med. 2000;108(Supl 4a):S99-S103.

4. Alexander JA, Hunt LW, Patel AM. Prevalence, pathophysiology, and treatment of patients with asthma and gastroesophageal reflux disease. Mayo Clinic Proc. 2000;75(10):1055-63.

5. Harding SM, Sontag SJ. Asthma and gastroesophageal reflux. Am J Gastroenterol. 2000;95(Supl 8):S23-S32.

6. Malagelada JR. Review article: supra-oesophageal manifestations of gastro-oesophageal reflux disease. Aliment Pharmacol Ther. 2004;19 (Supl 1):S43-S8.

7. Mansfield LE, Stein M. Gastroesophageal reflux and asthma: a possible reflex mechanism. Ann Allergy. 1978;41(4):224-6.

8. Tuchman DN, Boyle JT, Pack Al, Scwartz J, Kokonos M, Spitzer AR, et al. Comparisons of airway responses following tracheal and esophageal acidification in the cat. Gastroenterology 1984;87:872-9.
9. Harding SM, Guzzo MR, Richter JE. 24-h esophageal pH testing in asthmatics: Respiratory Symptom Correlation with Esophageal Acid Events. Chest. 1999;115(3):654-9.

10. Theodoropoulos DS, Pecoraro DL, Efstratiadis SE. The association of gastroesophageal reflux disease with asthma and chronic cough in the adult. Am J Respir Med. 2002;1(2):133-46.

11. Field SK, Sutherland LR. Does medical antireflux therapy asthma in asthmatics with gastroesophageal reflux?: a critical review of the literature. Chest. 1998;114(1):275-83.

12. Field SK. Gastroesophageal reflux and asthma: can the paradox be explained? Can Respir J. 2000;7(2):167-76.

13. Gibson P, Henry R, Coughlan J. The effect of treatment for gastroesophageal reflux on asthma in adults end children. Review [latest version 10 Feb]. In: Cochrane Library. Oxford: Update Software;1999.

14. Sociedade Brasileira de Pneumologia e Tisiologia. Diretrizes para Testes de Função Pulmonar. J Pneumol. 2002;28(3):1-221.

15. Johnson LF, Demeester TR. Twenty-four-hour pH monitoring of the distal esophagus. A quantitative measure of gastroesophageal reflux. Am J Gastroenterol. 1974;62(4):325-32

16. Harding SM, Richter JE, Guzzo MR, Schan CA, Alexander RW, Bradley LA. Asthma and gastroesophageal reflux: acid suppressive therapy improves asthma outcome. Am J Med. 1996;100(4):395-405.

17. Fernandes ALG, Oliveira MA. Avaliação de qualidade de vida nas asma. J Pneumol. 1997;23(3):148-52.

18. Juniper EF, Guyatt GH, Epstein RS, Ferrie JP, Jaeschke R, Hiller TK. Evaluation of impairment of health related quality of life in asthma: development of a questionnaire for use in clinical trials. Thorax. 1992;47(2):76-83.

19. Kiljander TO, Salomaa ER, Hietanen EK, Terho EO. Gastroesophageal reflux in asthmatics: a double-blind, placebo-controlled, crossover study with omeprazole. Chest 1999;116(5):1257-64.

20. Littner MR, Leung FW, Ballard ED 2nd, Huang B, Samra NK; Lansoprazole Asthma Study Group. Effects of 24 weeks of lansoprazole therapy on asthma symptoms, exacerbations, quality of life, and pulmonary function in adult asthmatic patients with acid reflux symptoms. Chest. 2005;128(3):1128-35.

21. Jiang SP, Liang RY, Zeng ZY, Liu QL, Liang YK, Li JG. Effects of antireflux treatment on bronchial hyper-responsiveness and lung function in asthmatic patients with gastroesophageal reflux disease. World J Gastroenterol 2003;9(5):1123-5.

22. Harding SM. Gastroesophageal reflux: a potential asthma trigger. Immunol Allergy Clin North Am. 2005;25(1):131-48.

23. Maev IV Viuchnova ES, Balashova NN, Shchekina Ml. Use of omeprazole and esomeprazole in patients suffering from bronchial asthma with associated gastroesophageal reflux disease. Eksp Klin Gastroenterol 2003; 116(3):26-31.

24. Ribeiro 1, Cardoso PFG, Hetzel JL, Camargo JJP, Felicetti $\mathrm{JC}$. Esofagomanometria e pHmetria esofagiana ambulatorial de 24 horas nos pacientes com asma com e sem sintomas digestivos associados. J Pneumol 2000;26(Supl 3):S16.

25. Fouad YM, Katz PO, Hatlebakk JG, Castell DO. Ineffective Esophageal Motility: the Most Common Motility Abnormality in Patients with GERD-Associated Respiratory Symptoms. Am J Gastroenterol 1999;94(6):1464-7. 
Avaliação da resposta de pacientes asmáticos com refluxo gastroesofágico após terapia com pantoprazol: estudo prospectivo, randomizado, duplo cego e placebo-controlado

26. Sontag SJ, OConnell S, Khandelwal S, Greenlee H, Schnell T, Nemchausky B, et al. Asthmatics with gastroesophageal reflux: long term results of a randomized trial of medical and surgical antireflux therapies. Am J Gastroenterol. 2003 98(5):987-99.
27. Boeree MJ, Peters FT, Postma DS, Kleibeuker JH. No effects of high-dose omeprazole in patients with severe airway hyperresponsiveness and (a)symptomatic gastro-oesophageal reflux. Eur Respir J. 1998;11(5):1070-4. 